\title{
A comparative study on shared-use medicines in Tibetan and Chinese medicine
}

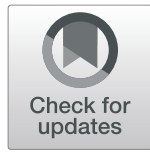

Ming-ming Zhao ${ }^{1,2}$, Ke-ru Wang ${ }^{1}$, Rui Gu ${ }^{1 *}$ (D) and Shi-hong Zhong ${ }^{3}$

\begin{abstract}
Background: Tibetan medicine (TM) and traditional Chinese medicine (TCM) are two independent traditional medical systems. Due to geographical factors, the development of Tibetan medicinal theory is relatively independent, but there are still many shared-use medicines in TM and TCM. However, a thorough and comparative study on those medicines is still absent. This study listed shared-use medicines by TM and TCM and analyzed the similarities and dissimilarities of these two medical systems. This paper also aimed to understand mutual influences like the shared history of TM and TCM and to roughly outline the exchanging process between them.

Methods: Shared-use medicines in TM and TCM were listed alphabetically. Information on the scientific name, material name, medicinal parts, and medical efficacy were extracted from publications. Shared-use medicines were grouped according to medicinal properties and medicinal parts used by TM and TCM. The historical origin and current status of clinical prescriptions of shared-use medicines were analyzed.

Results: A total of 136 shared-use medicines in TM and TCM were listed. Shared-use medicines that were used for a similar purpose in TM and TCM accounted for $14 \%$ of the total, while those used for different purposes accounted for $49 \%$ of the total, with some of the latter being commonly used in TCM. Shared-use medicinal herbs that originated from both Tibetan and Han regions accounted for $49 \%$ of the total, and those that were imported from South Asia and Southeast Asia were frequently observed in TM.

Conclusion: Owing to its unique geographical location and cultural diversity, the Tibetan region played a role as a development cradle for various traditional medicinal theories and knowledge. Medicinal knowledge was exchanged between TM and TCM during their parallel independent growth. Shared-use medicines in TM and TCM were mostly determined by flora similarity and medicinal trade, and they marked significant differences in their medicinal properties. However, medicines that were used for similar purposes in TM and TCM presented obvious commercial medicinal characteristic as well as the same chemical profile. The Tibetan region not only provided medicinal usage knowledge of TCM, but also served as a supply of medicinal resources attributing to "high altitude" locations.
\end{abstract}

Keywords: Tibetan medicine, Traditional Chinese medicine, Shared-use medicines, Comparative study

\section{Background}

Tibetan medicine (TM) is rich in medicinal resources, approximately $80 \%$ of which are produced in the Qinghai-Tibet Plateau, with significant ethnic culture and regional biological distinctness, which is reflected in it being a culturally traditional medicine. Tibetan medicine presents obvious characteristics of national culture and distinct ecological-geographic conditions [1]. It is generally believed in medical documents that during the

\footnotetext{
* Correspondence: 664893924@qq.com

${ }^{1}$ Chengdu University of Traditional Chinese Medicine, No. 1166, Liu-tai Road, Wenjiang District, Chengdu 611173, China

Full list of author information is available at the end of the article
}

inheritance and development process, TM has continuously acquired knowledge and absorbed beneficial ingredients from Chinese traditional medicine (TCM), Indian Ayurveda medicine, and Arabic medicine. Earlier research on the history of Tibetan medicine [2] showed that Chinese and Tibetan medicines were widely exchanged during the Tubo period, while there are few documents about medical communication occurring between Han and Tibetan people at a later period. Princess Wencheng of the early Tang Dynasty brought "a hundred prescriptions for the treatment of four hundred and four diseases, five kinds of diagnostic methods,

(c) The Author(s). 2019 Open Access This article is distributed under the terms of the Creative Commons Attribution 4.0 International License (http://creativecommons.org/licenses/by/4.0/), which permits unrestricted use, distribution, and 
six kinds of medical devices, and four kinds of theory on medicine..." to Tibet, following her marriage with King Songtsan Gambo of Tibet. A Han doctor translated them into the Medical Encyclopedia, and later, traditional Chinese medicine (TCM) practitioner Han Wenhai contributed Small Sporadic External Therapy and Fearless Weapons coauthored with Scorpion and Persian Doctors during the period of Chi Dezuzan (704-745). Princess Jincheng dedicated a variety of medical books, such as Jumbo Drugs by Han doctor Zhang Song and Human Body Torso translated by doctor Zambia Laha from Yutian, in the Western Region, to the Tibetan king. During the period of Akamatsu Dezan, Yuewang Drug Clinic was translated into the Tibetan language, and three famous Chinese medical practitioners, including Dongsong Gangwa of the Tang Dynasty, were recruited. At the end of the eighth century, Yutuo Yuandan Gongbu was sent by the Tibetan King to the mainland, such as Wutai mountain, to study TCM [3].

Based on the above historical literature, many scholars believe that Tibetan medicine has been largely influenced by traditional Chinese medicine during its development. An earlier article [4] revealed that there were many connections in the historical background, theoretical system, and application of prescriptions between TM and TCM, and TM possesses its own unique features in addition to what it shares in common with TCM regarding medicinal properties, pharmacology, clinical medical practices, prescriptions, and patent medicines. However, we still do not know much about the similarity or dissimilarity of the two traditional medicinal systems, and a thorough study on shared-use medicines in TM and TCM is still absent. In view of this, the main goals of the present paper are to systematically list shared-use medicines in TM and TCM, evaluate the differences of the medicinal parts and medical efficacies, analyze the mutual influence of history and the exchanging process of the two medical systems, and discuss the historical and cultural background leading to those shared-use medicines.

\section{Methods}

TCM use a total of 12,807 [5] different types of medicine, and TM use 3105 [6] species according to documentation. However, comprehensive statistics on these total varieties cannot reflect the clinical status of TM and TCM. Luckily, earlier, Zhong Guoyue compiled 502 medicinal materials that are used in the Tibetan medicine prescriptions. The present paper listed commonly used Tibetan medicines based on his study, including publications such as the Ministry of Health of the People's Republic of China - Tibetan Medicine, Book 1 [7] and the Tibetan Medicine Standards for Six Provinces [8]. According to The Collection of Chinese Herbal
Medicines [9], shared-use medicines, including animal, plant, mineral, and fungi resources, were documented alphabetically. Information on the scientific name, medicinal parts, and medical efficacy was extracted from publications, such as Chinese Pharmacopoeia, Book 1 (2015 edition) [10], The Collection of Chinese Herbal Medicines [9], Chinese Medicine Dictionary [11], Ministry of Health of the People's Republic of China - Tibetan Medicine, Book 1 [7], Tibetan Medicine Standards for Six Provinces [8], The Four-Part Medical Classics [11], Crystal Beads [12], Tibetan Medicine Chronicles [13], Chinese Materia Medica (Tibetan Medicine) [14], Tibetan medicine in China [15], and Tibetan Herbals in China [16], and was pooled together. Information on the family and genus was analyzed based on Flora Reipublicae Popularis Sinica [17], and Higher Plants of China [18]. The shared-use medicines recorded by Chinese Pharmacopoeia, Book 1 (2015 edition) [10] and the Medical Standards of the Ministry of Health of the People's Republic of China - Tibetan Medicine, Book 1 [7] were marked in this paper.

According to the medicinal property, the shared-use medicines in TM and TCM were divided into five categories: classes I, II, III, IV, and V. The shared-use medicines with the same purpose fell into class I; the shared-use medicines with medicinal usage by TM basically covering that by TCM fell into class II; the shareduse medicines with medicinal usage by TCM basically covering that by TM fell into class III; the shared-use medicines that were used for different purposes fell into class IV; and the shared-use medicines with partial overlapping medical effects in the two medicinal system fell into class $\mathrm{V}$.

According to the medicinal part differences, the shared-use medicines were divided into five types: classes $A, B, C, D$, and $E$. The shared-use medicines with same medicinal parts in TM and TCM fell into class A; the shared-use medicines with medicinal parts in TM covering that in TCM fell into class B; the shared-use medicines with medicinal parts in TCM covering that in TM fell into class $C$; the shared-use medicines with totally different medicinal parts in the two medical systems fell into class D; and the shared-use medicines with partial overlapping medicinal parts in the two medicinal system fell into class $\mathrm{E}$. The above information were given in Tables 1, 2, 3, 4 and 5.

Based on the plant distribution recorded in the Flora Reipublicae Popularis Sinica and other research on medicinal materials, the origins of the shared-use medicines could be roughly divided into the following five types: the first category included varieties that were distributed in both the Tibetan region and in the areas that were mainly covered by the Central Plains throughout history; the second category included varieties that were mainly 


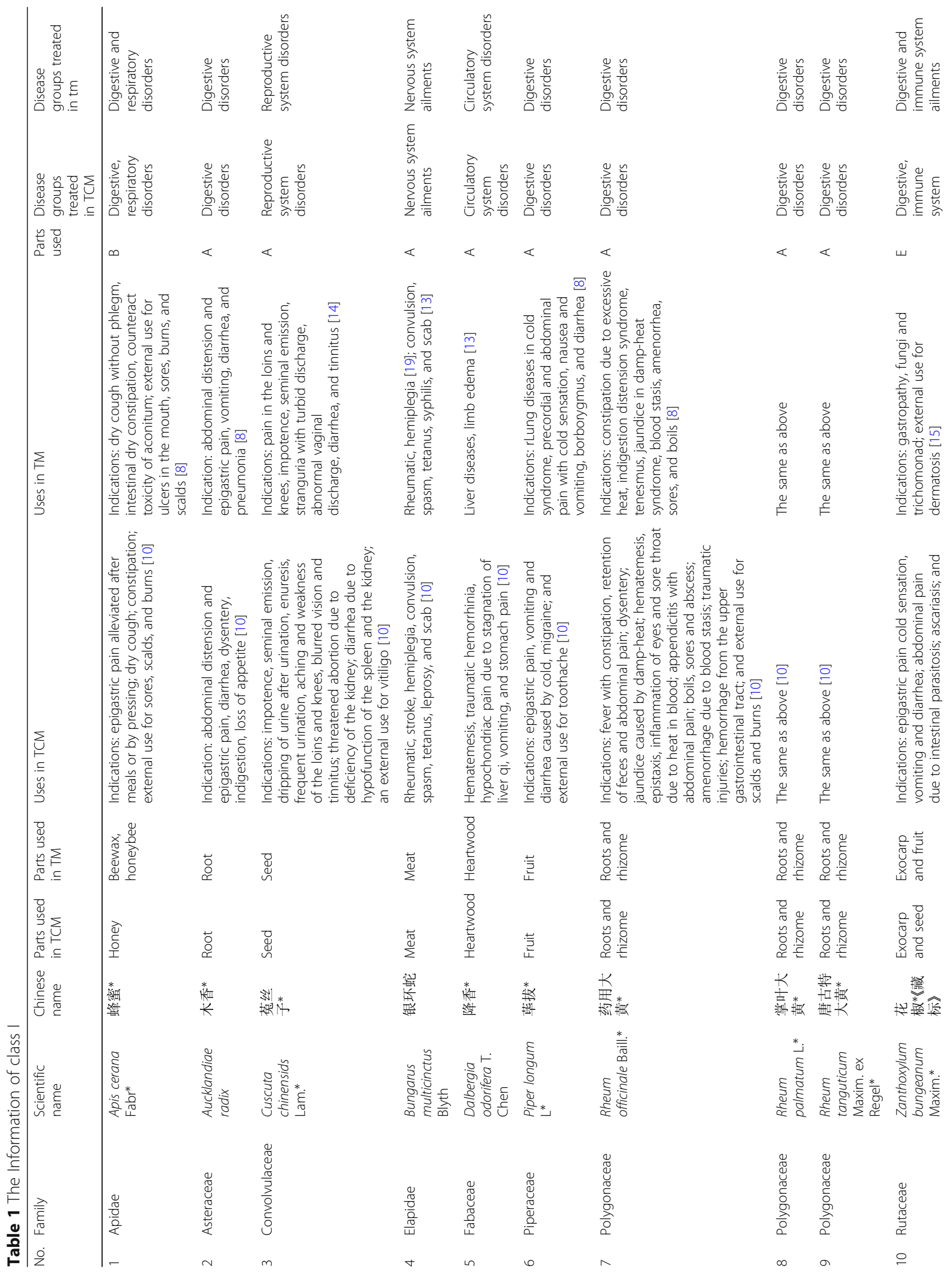




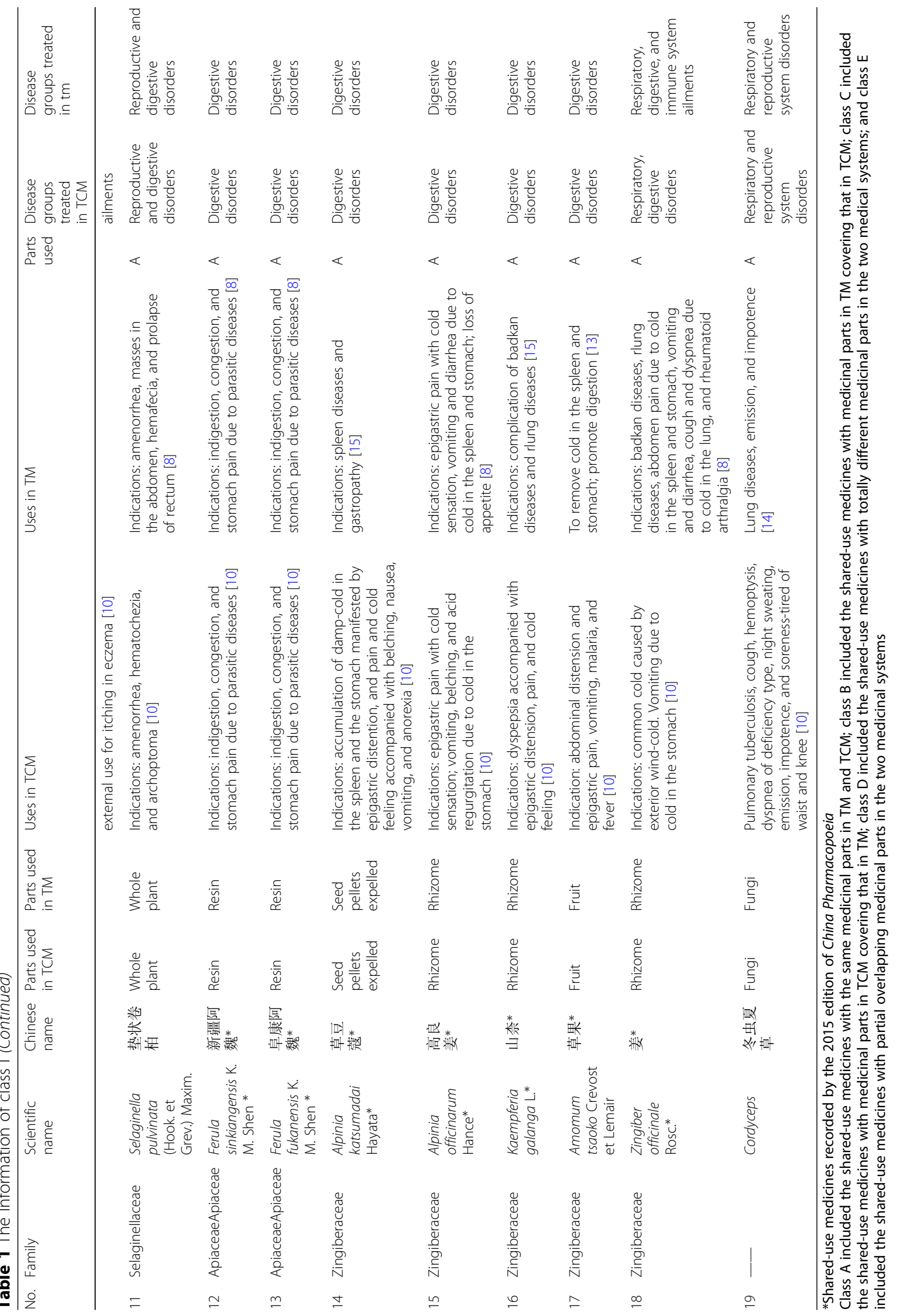




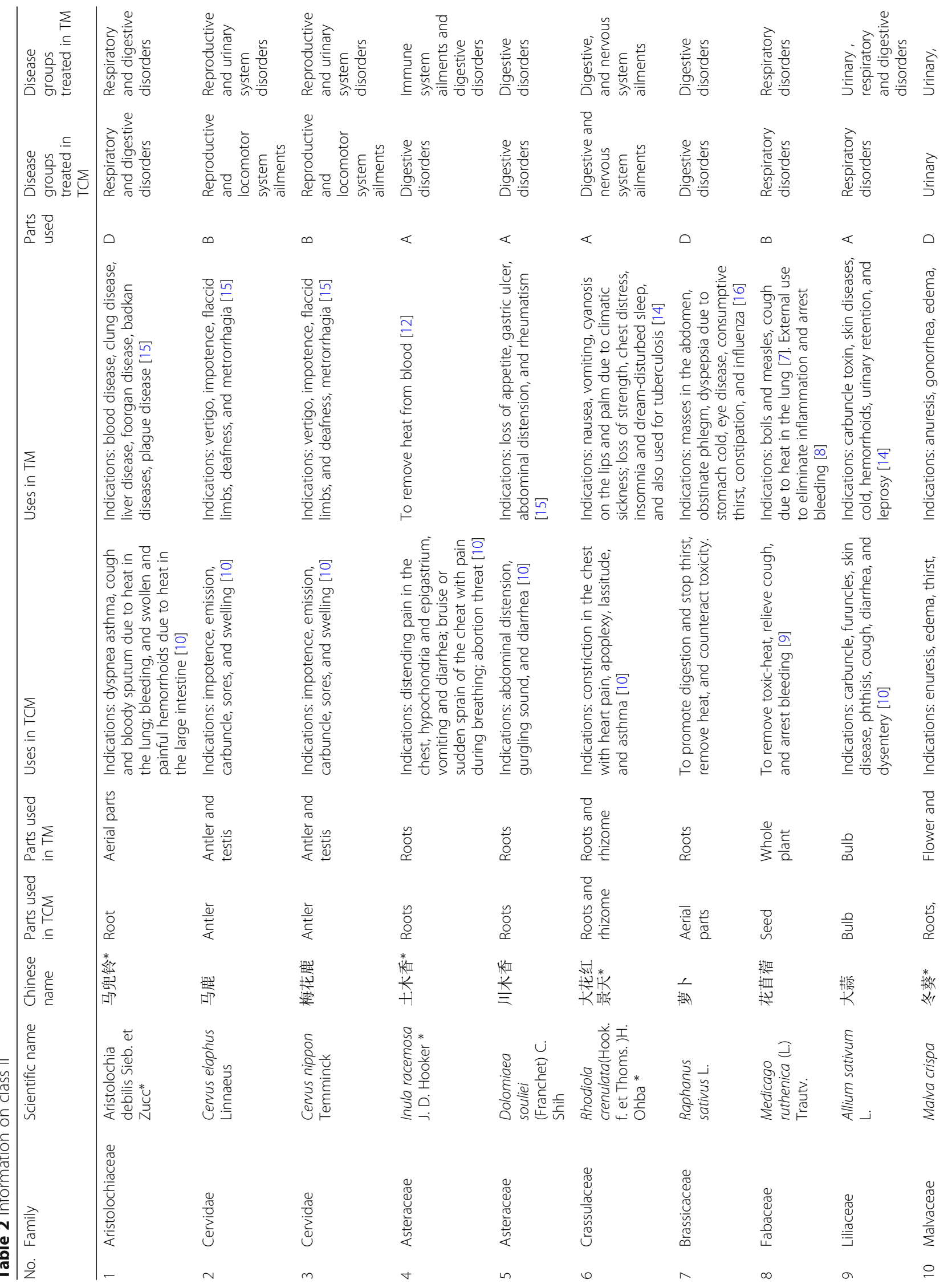




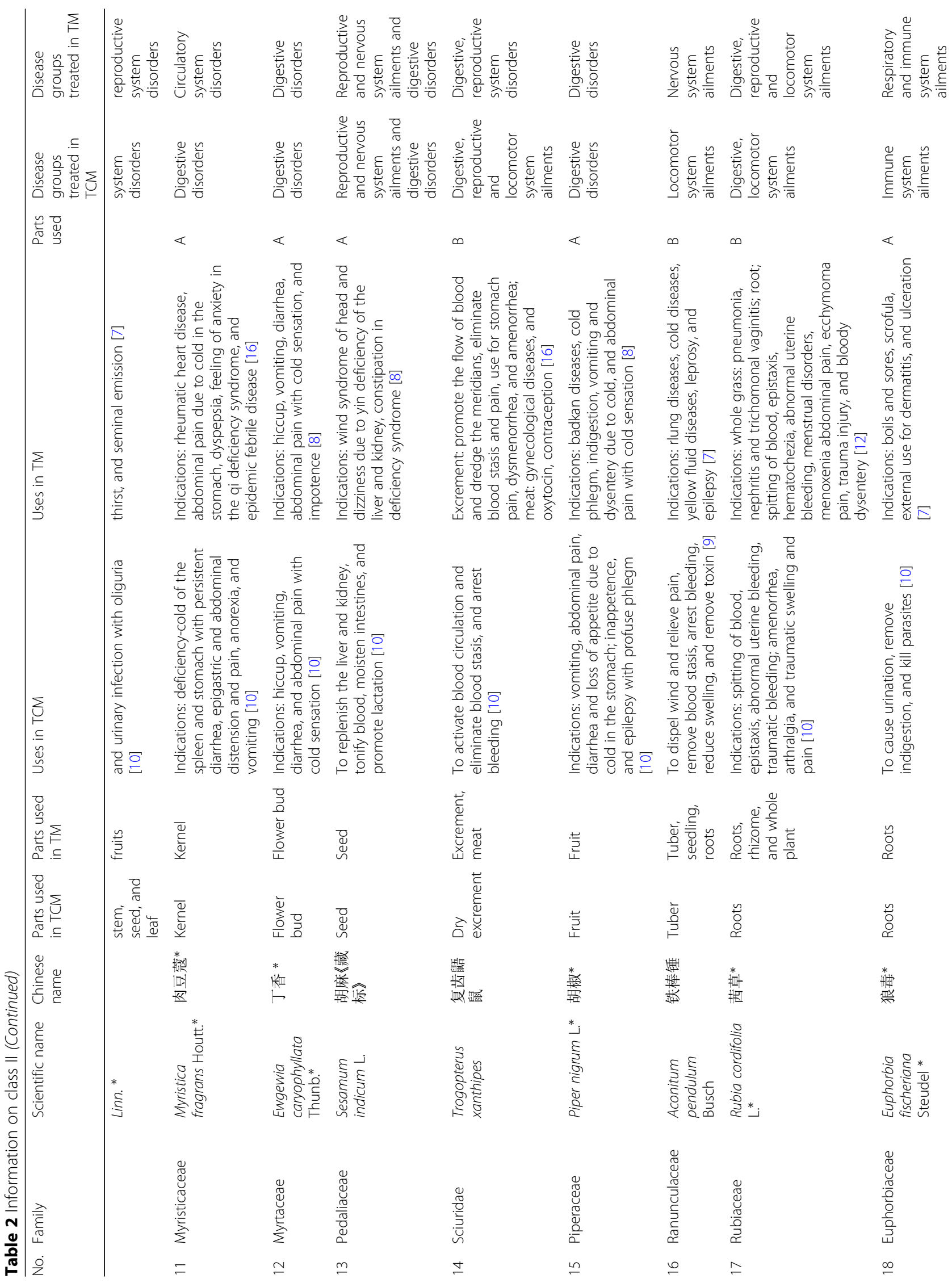




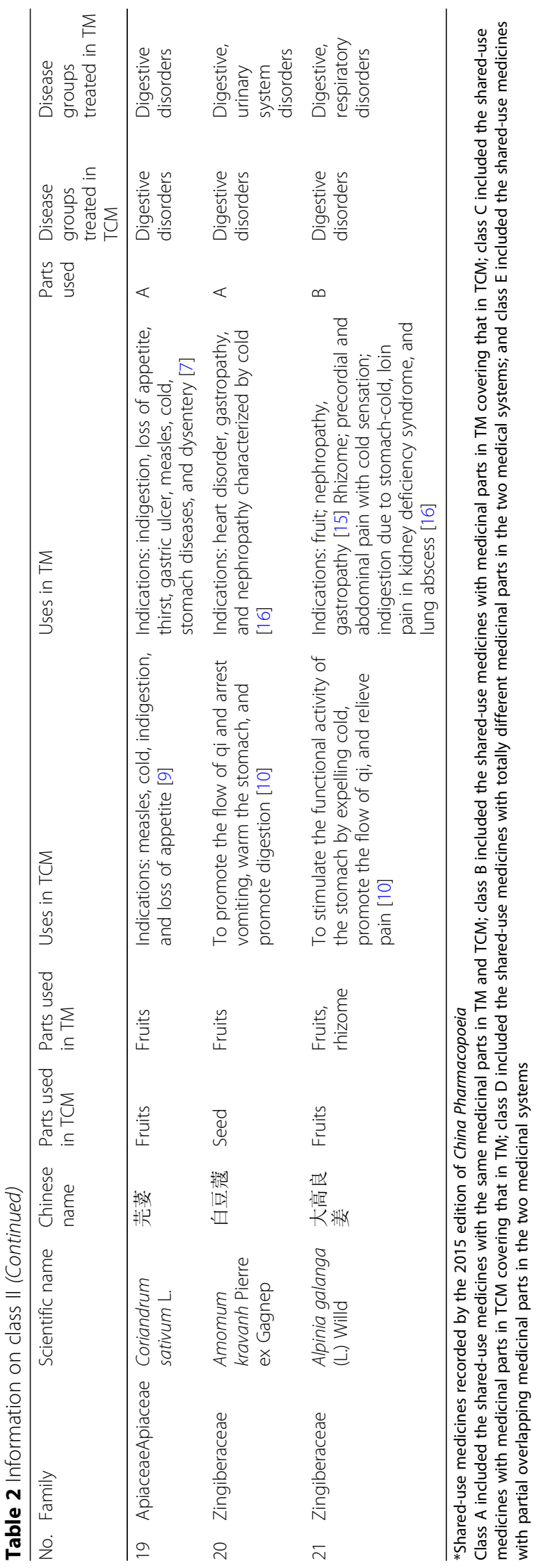




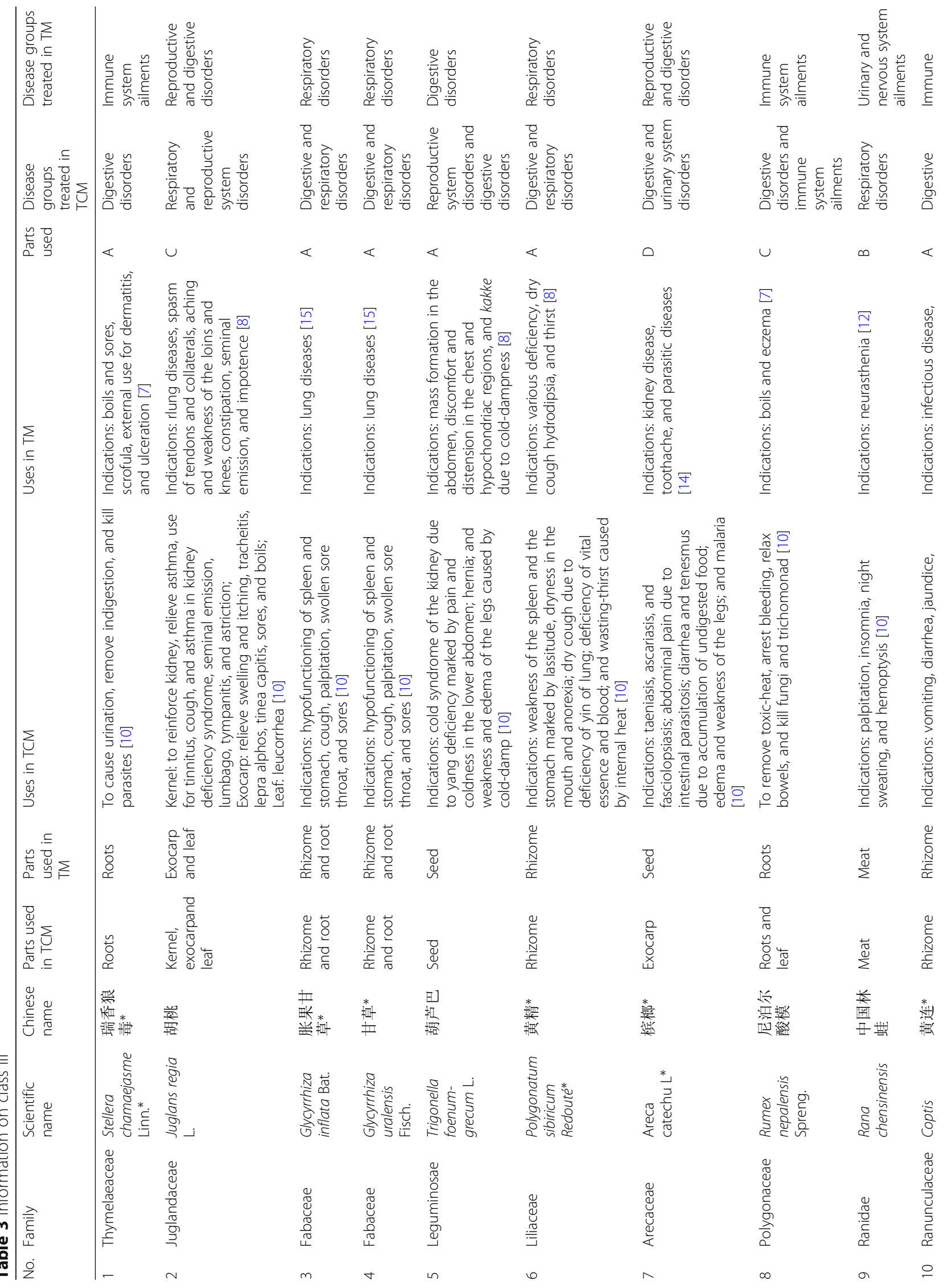




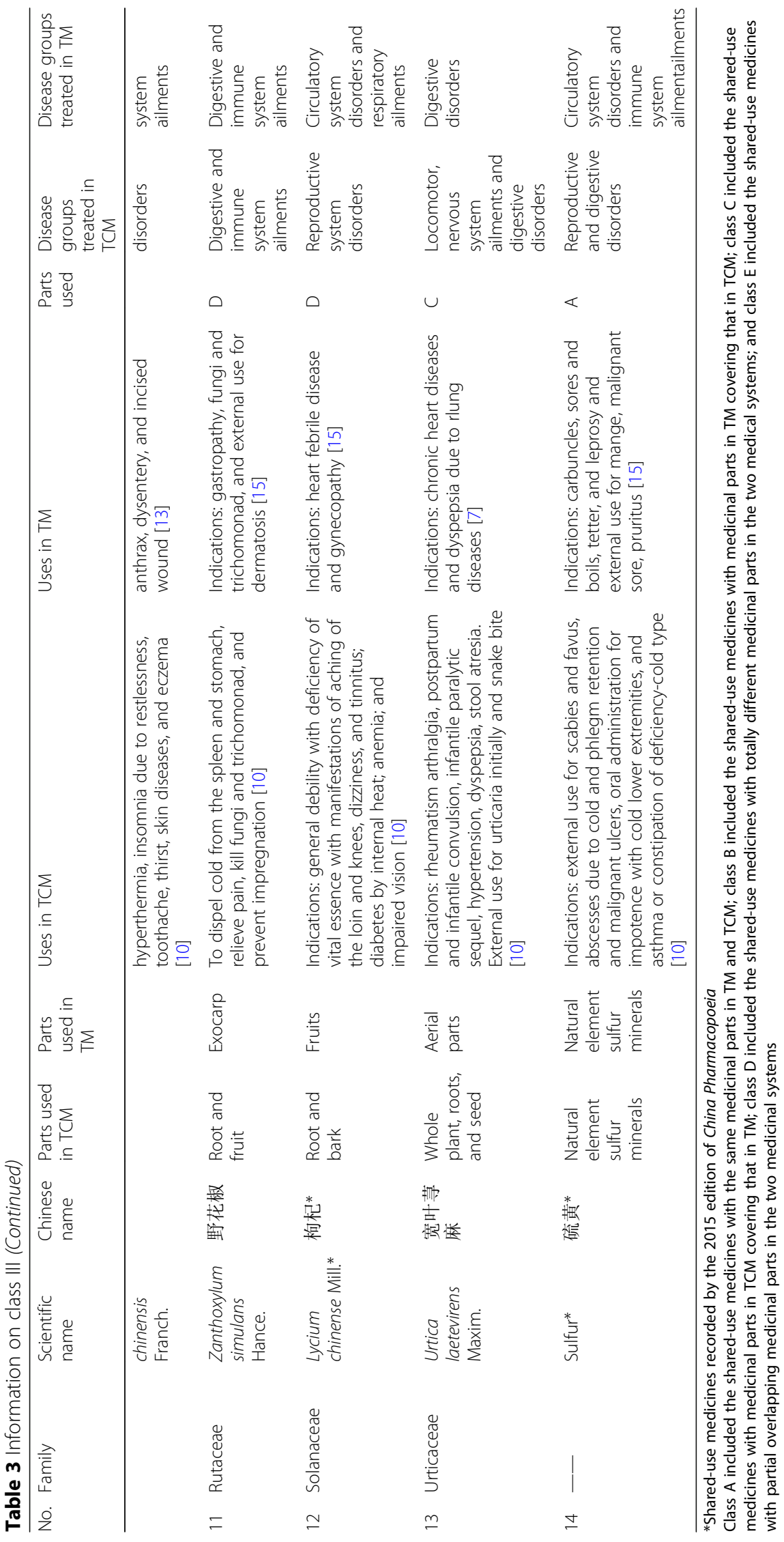




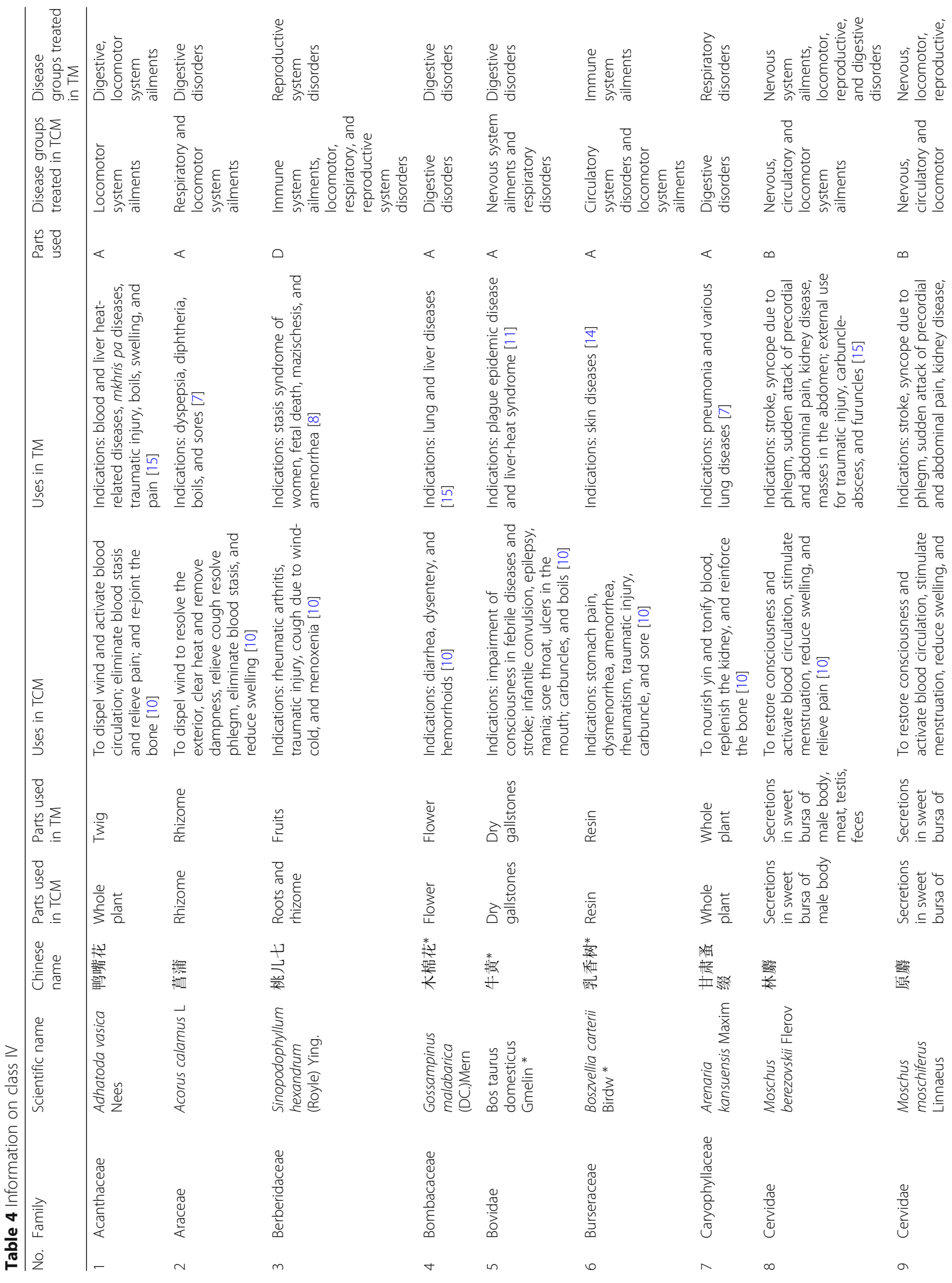




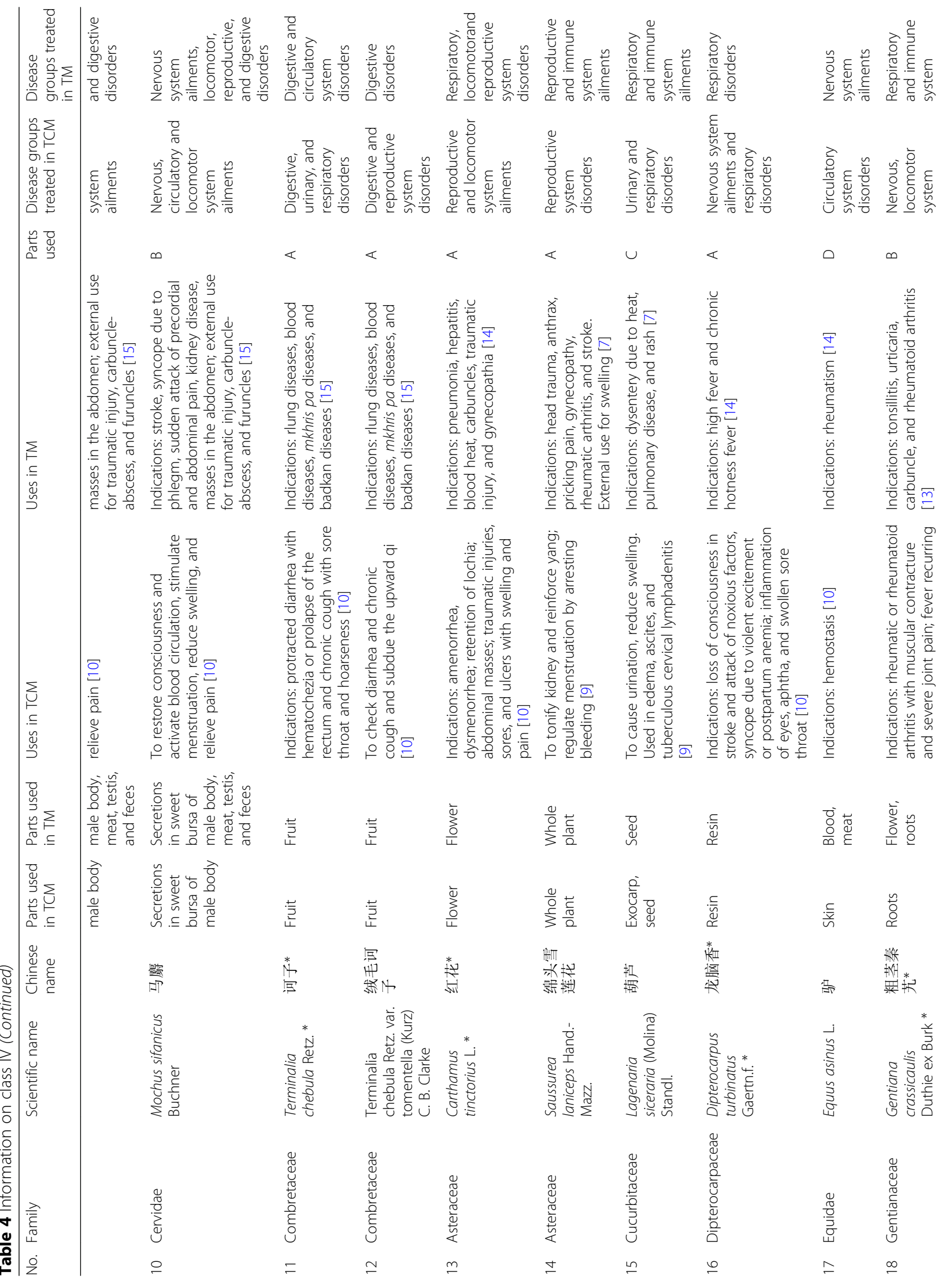




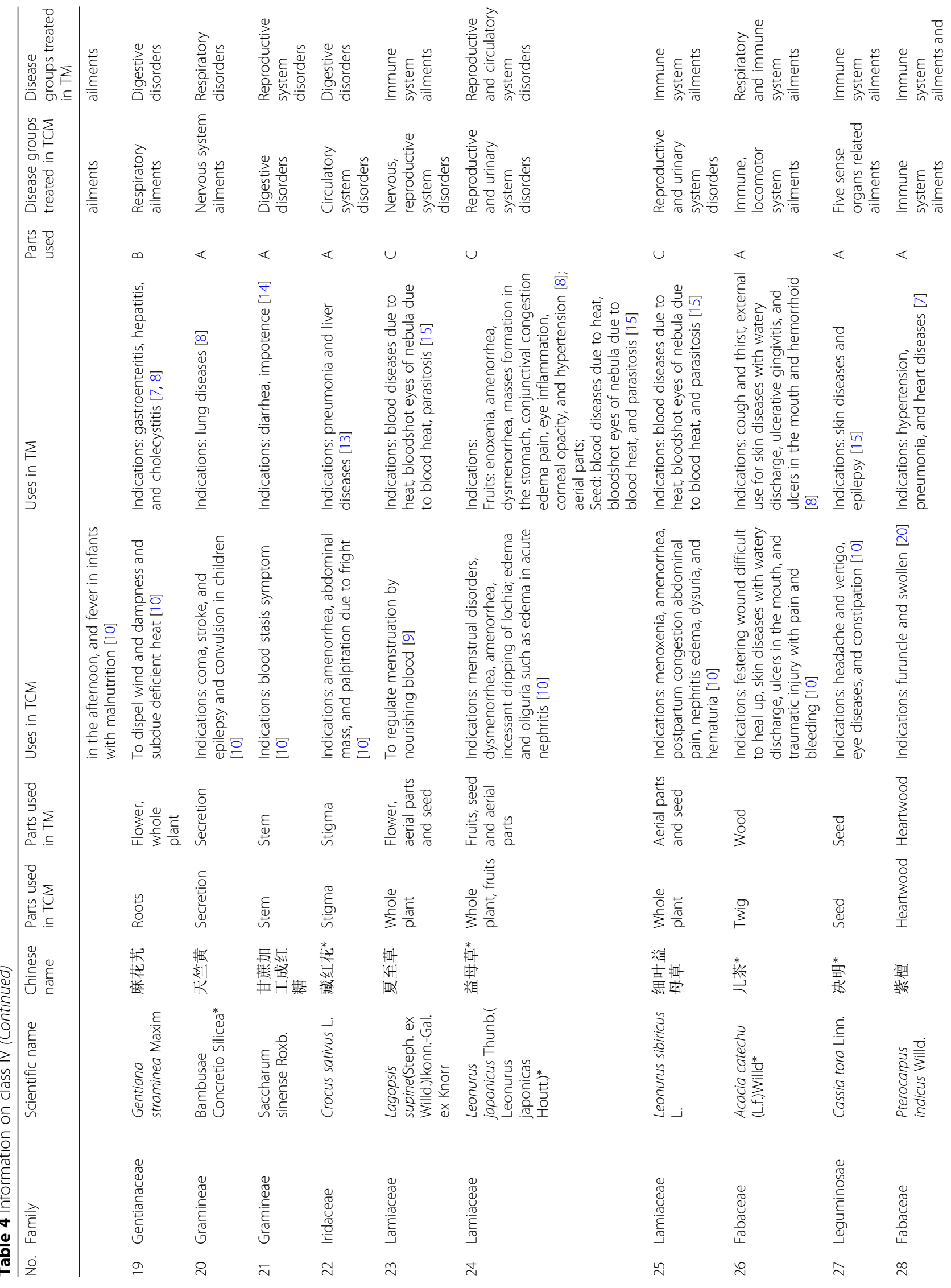




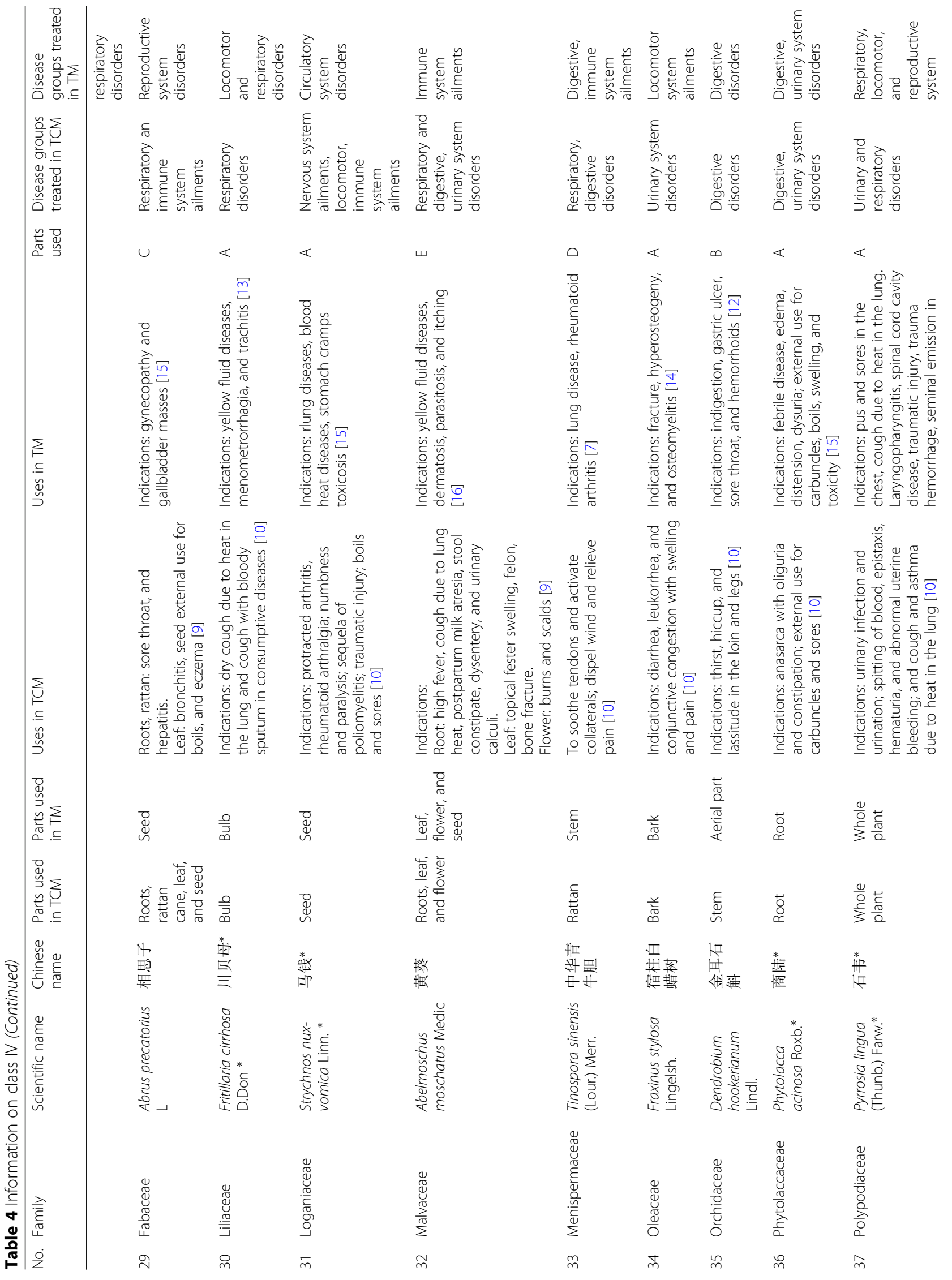




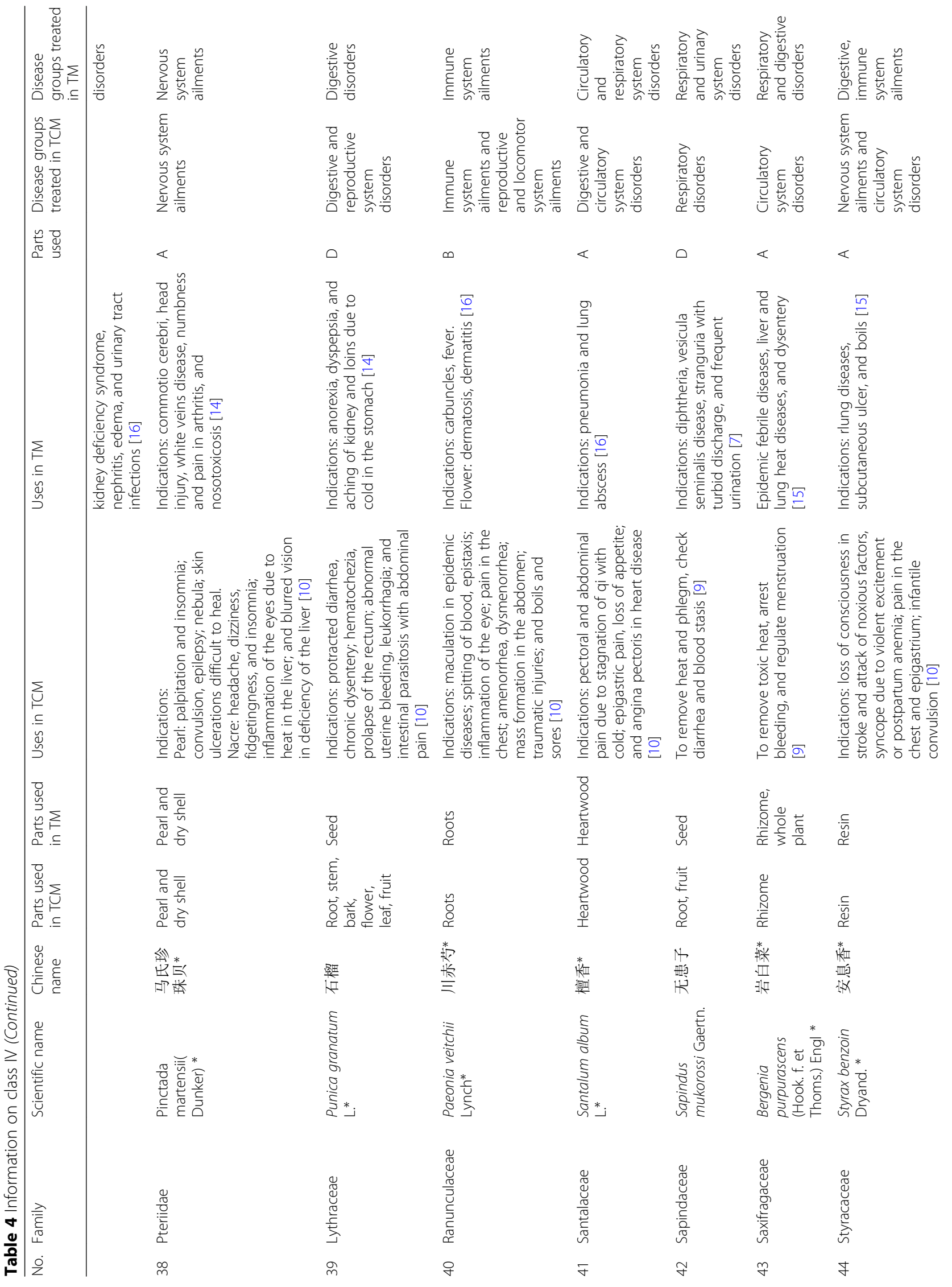




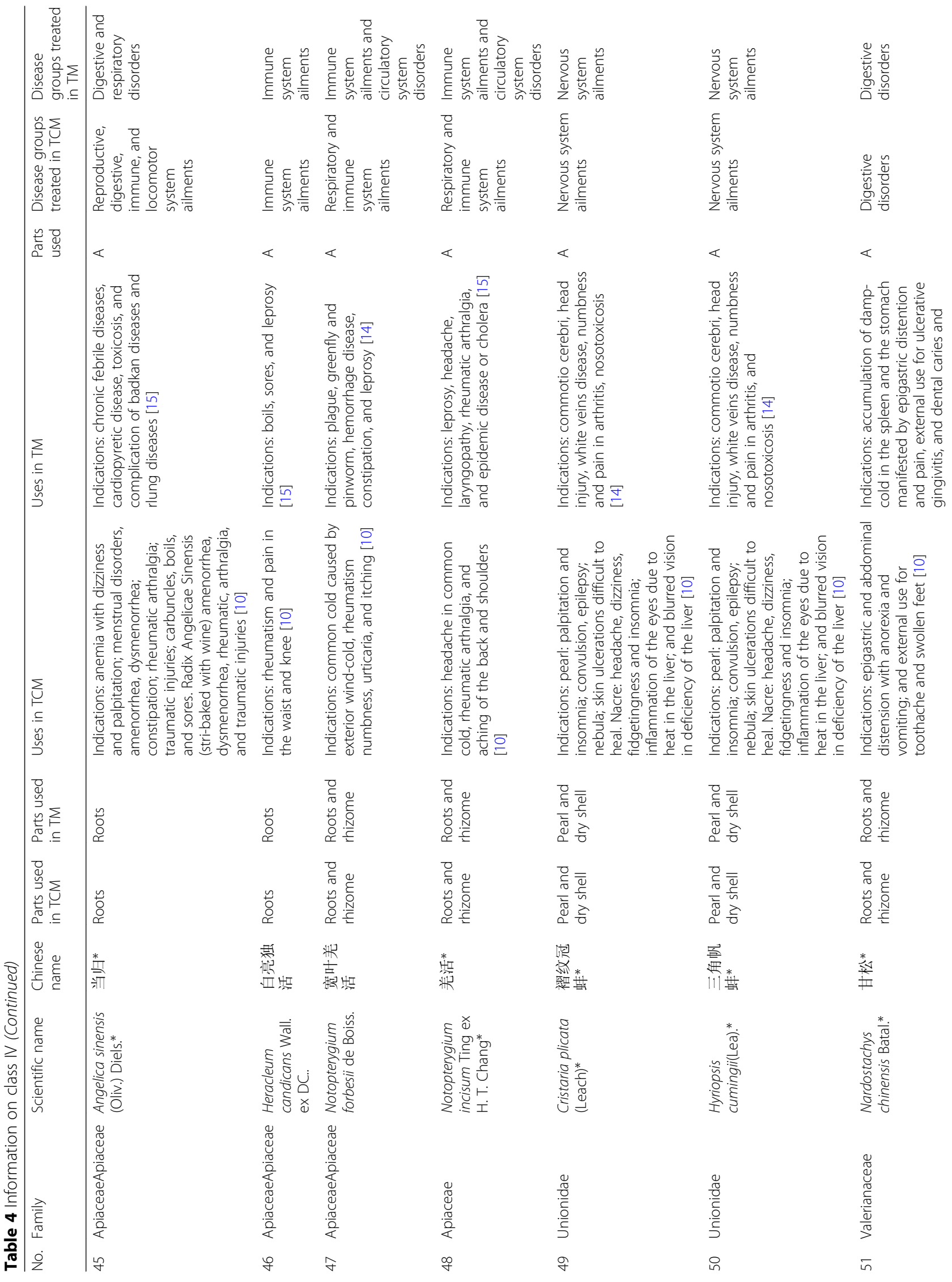




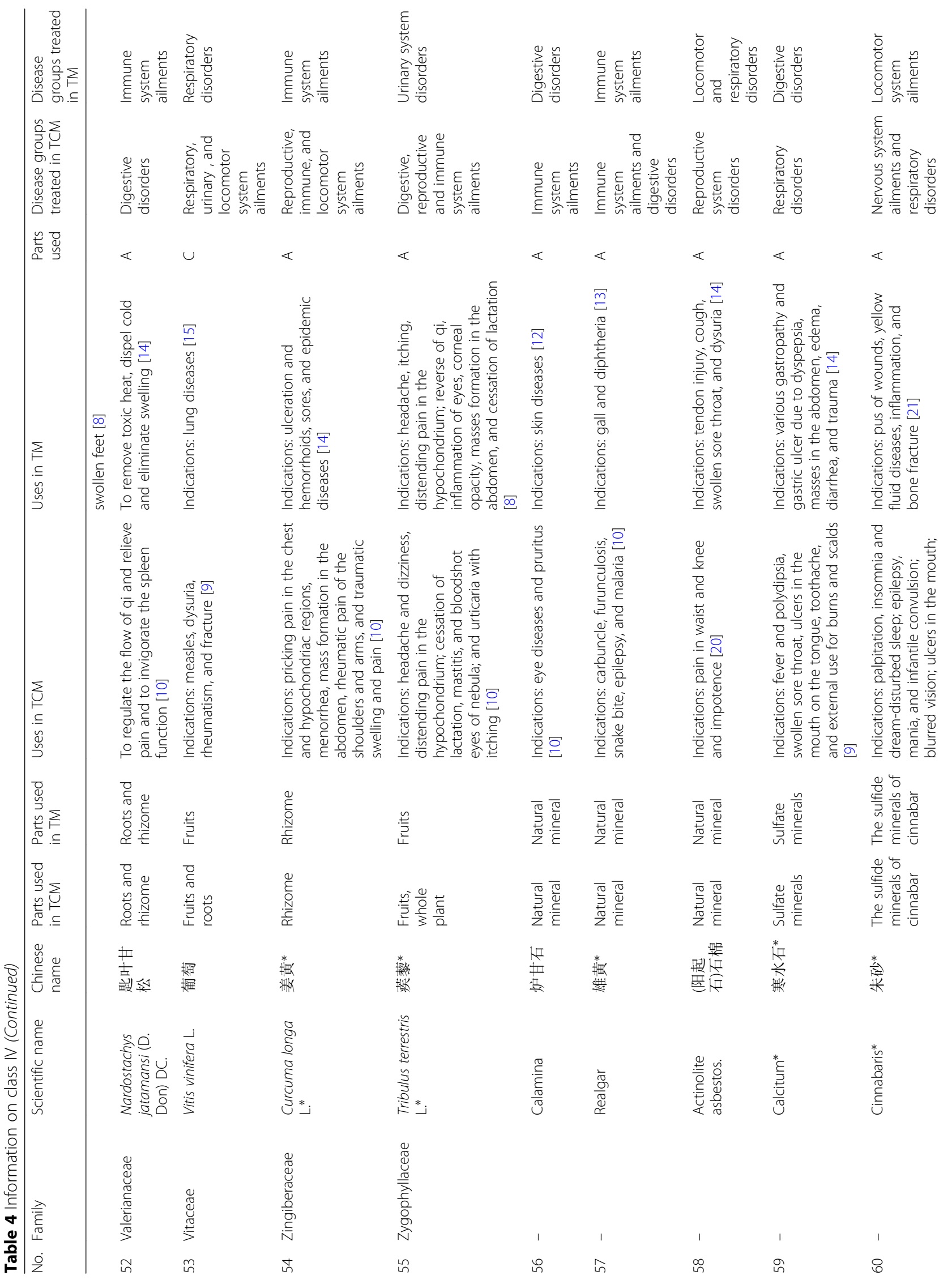




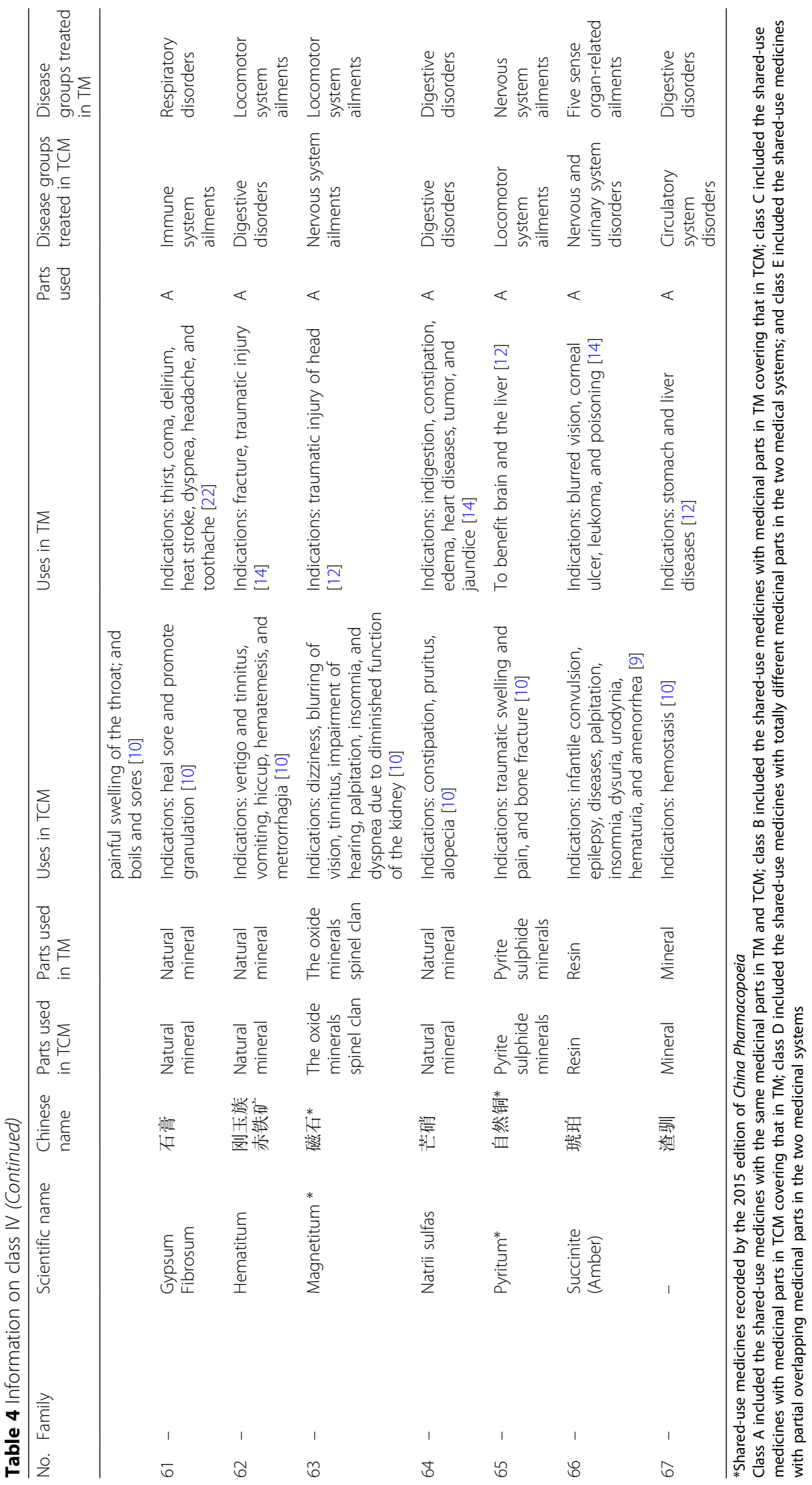




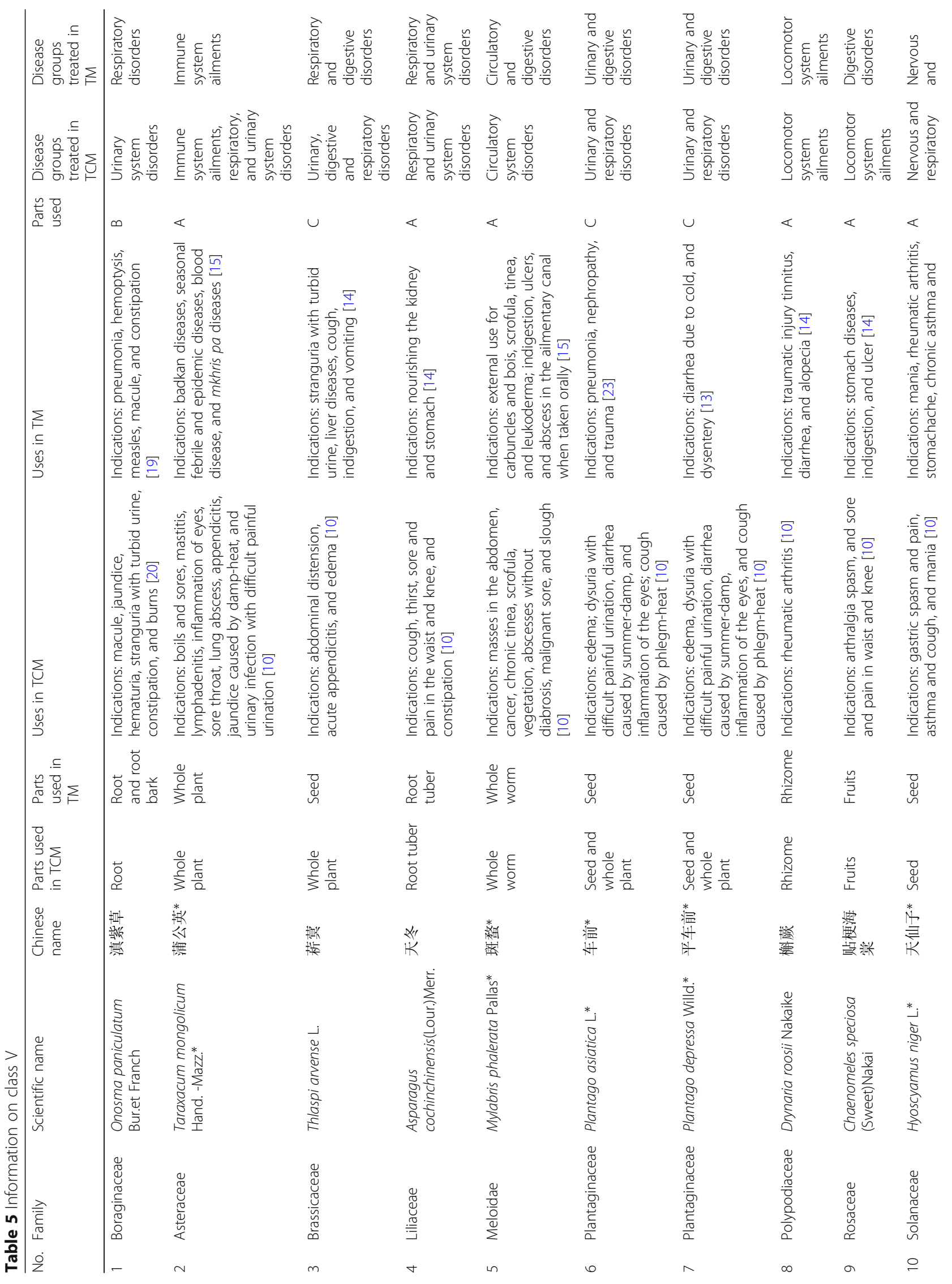




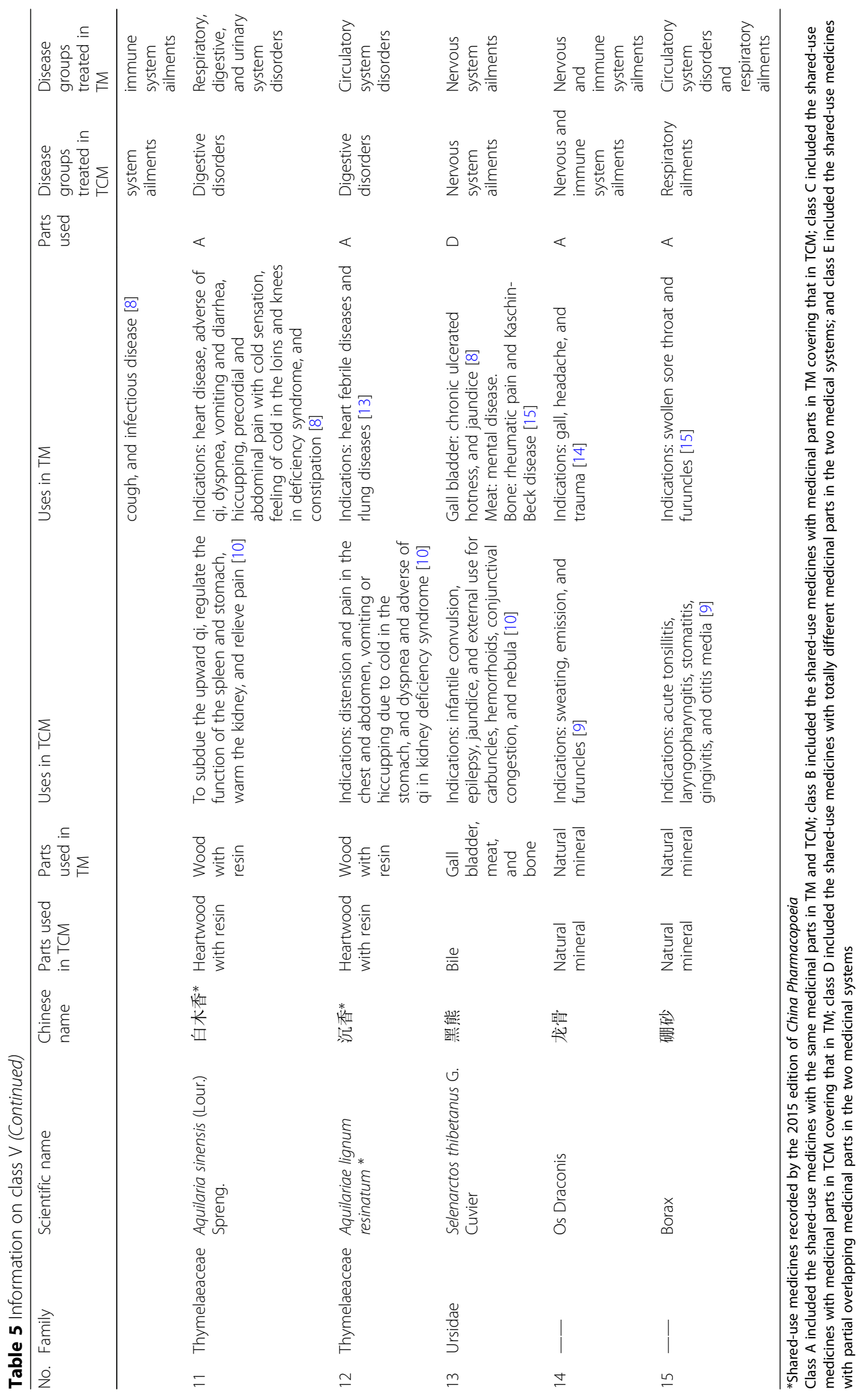


Table 6 Number of species of shared-use medicines grouped by used parts and efficacy classification

\begin{tabular}{llllllll}
\hline Classification & A & B & C & D & E & Total & Percentage of different efficacy groups to the total \\
\hline I II & 17 & 1 & 0 & 0 & 1 & 19 & $14 \%$ \\
III & 11 & 7 & 0 & 3 & 0 & 21 & $15 \%$ \\
IV & 7 & 1 & 3 & 3 & 0 & 14 & $10 \%$ \\
V & 48 & 7 & 6 & 5 & 1 & 67 & $49 \%$ \\
Total & 10 & 1 & 3 & 1 & 0 & 15 & $11 \%$ \\
Percentage of different used parts grouped in total & $98 \%$ & $13 \%$ & $9 \%$ & $9 \%$ & $1 \%$ & & \\
\hline
\end{tabular}

produced in the Tibetan Plateau and its surrounding areas; the third category included varieties that were imported from South Asia, Southeast Asia, and Western Regions; the fourth category included the varieties that were generated inland and were traded in the Tibetan region; and the last category included the varieties that were mainly imported from the Han district. Detailed information was given in the Table 12 .

\section{Results}

The similarity of medicinal parts and the efficacy of shared-use varieties used in TM and TCM

A total of 136 species of shared-use medicines that are used in prescriptions of TM and TCM were listed, and detailed information was given in Table 6 and Fig. . 1.

As shown in the above chart, nearly $50 \%$ of the shared-use medicines are used for different purposes in TM and TCM (class IV), whereas shared-use medicines with the same utilization accounted for $14 \%$ (class I), which indicated that extremely different knowledge on medicine usage existed between TM and TCM.

The proportion of shared-use medicines with similar utilizations (classes I, II, III, and V) reached 50\%, which suggested a medicinal knowledge exchange between the two medical systems. For example, a number of 49 species from the inland area and 7 species from Sichuan province were recorded by the classical Tibetan medical

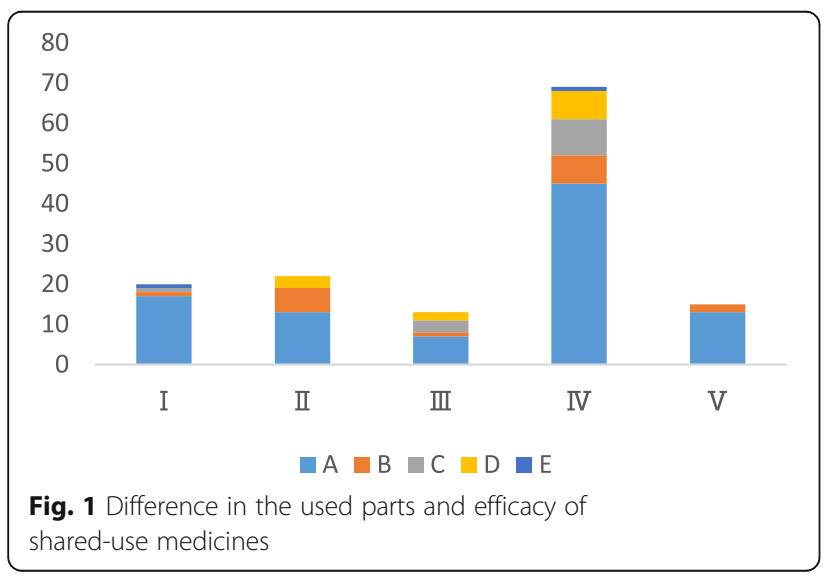

book Crystal Beads [12]. However, both TM and TCM have immense diversity in their medicinal parts, and as the medical effects of one of them cover that of the other, the medicinal parts tend to be more diverse.

\section{The origins of shared-use varieties used in TM and TCM}

According to Tables 1, 2, 3, 4 and 5, the origins of shared-use medicines used by TM and TCM were documented in Table 7.

As shown in Table 7, the shared-use medicines that are distributed in both the Han inland and the Tibetan region accounted for the majority of the total (49\%, which is the first category). Thirty-five percent of the total were imported by TM from the non-Han area through trade routes (the third category and the fourth category). Fifteen percent of the total depended on import from both the Tibetan and Han regions. Interestingly, varieties that were produced in the Tibetan region exclusively (the second category) were far more common than those that were imported from Han inland (the last category), which supported the fact that the QinghaiTibet Plateau also serves as the supplier for medicinal resources of TCM. The proportion of imported varieties in class I was significantly higher than that in the other groups, indicating that the shared-use medicines with the same efficacy were obviously affected by the extraterritorial medical systems. The proportion of shared-use medicines that were produced exclusively in Tibet (the second category) and the shared-use medicines that were mainly traded in the Tibetan region (the fourth category) were higher than those of other origins in class IV, which suggested a connection and an obvious evidence of independent development of the TM and TCM systems.

The clinical application status of shared-use varieties used by TM and TCM

Since the prescription preparations are used to treat diseases in TM, the frequency of the utilization of shared-use medicines in prescription reflects its clinical application status. According to the statistical results of Tibetan medicines that are used, frequency was compiled by Zhong Guoyue [1], and the clinical application status 
Table 7 Number and percentage of different origins of shared-use medicines (classes I-V)

\begin{tabular}{llllllll}
\hline & I & II & III & IV & V & The total number of different origins & Percentage of different origins of shared-use medicines \\
\hline The first category & 6 & 12 & 8 & 28 & 13 & 67 & $49 \%$ \\
The second category & 2 & 2 & 0 & 12 & 0 & 16 & $12 \%$ \\
The third category & 6 & 4 & 1 & 8 & 1 & 20 & $15 \%$ \\
The fourth category & 3 & 3 & 3 & 18 & 1 & 28 & $20 \%$ \\
The fifth category & 2 & 0 & 2 & 1 & 0 & 5 & $4 \%$
\end{tabular}

The first category included varieties that distributed in both Tibetan region and the areas mainly controlled by the Central Plains regime in history; the second category included varieties that were mainly produced in the Tibetan Plateau and its surrounding areas; the third category included varieties that were imported from South Asia, Southeast Asia, and Western Regions; the fourth category included the varieties that were generated in the inland and that were traded in the Tibetan region; the last category included the varieties that were mainly imported from the Han district

of shared-use medicines was shown in the following Table 8.

TCM emphasizes that the use of medicine should be compatible according to clinical needs, so the importance of shared-use medicine can be reflected by its application frequency in clinical prescriptions. Earlier, Ping [24] et al. listed 10,000 prescriptions from the First Affiliated Hospital of Guangzhou and the University of Traditional Chinese Medicine and sorted out the top 50 commonly used medicinal items, among which there are only four that were shared-use in TM and YCM, as shown in Tables 8 and 9.

In Tables 8 and 9, the shared-use medicines occupied a far more important place in clinical prescriptions of TM than TCM. Among the medicinal materials that were used in more than 51 Tibetan clinical prescriptions, shared-use medicines in TM and TCM accounted for $73 \%$, and $90 \%$ of those were imported from South Asia, Southeast Asia, and Western regions. Of the top 10 medicinal materials in usage frequency, most were imported, except for musk and Zha-xun.

\section{Comparison of the therapeutic systems of shared-use medicines used in TM and TCM}

The present study has attempted to group the shareduse medicines according to the similar types of diseases that affect the same part of the body; data was extracted from Tables 1, 2, 3, 4 and 5, as shown in Table 10.

It can be easily observed from Table 10 that there was a high similarity between TM and TCM in treating digestive diseases, respiratory ailments, circulatory system diseases, and urogenital disorders. A large proportion of herbal aromatics viz., Dalbergia odorifera T. Chen, Resina ferulae, Kaempferia galanga L., Piper longum L, Amomum kravanh Pierre ex Gagnep, Ewgewia caryophyllata Thunb., Piper nigrum L., Myristica fragrans Houtt., Alpinia katsumadai Hayata, Alpinia officinarum Hance, and Alpinia galanga (L.) Willd were

Table 8 Shared-use medicines involved in TM preparations

\begin{tabular}{|c|c|c|}
\hline $\begin{array}{l}\text { Number of } \\
\text { preparations } \\
\text { involved }\end{array}$ & $\begin{array}{l}\text { Scientific name of the } \\
\text { shared-use medicines }\end{array}$ & $\begin{array}{l}\text { The number of shared-use medicines/ } \\
\text { The total number of medicinal } \\
\text { materials[1] (percentage composition) }\end{array}$ \\
\hline$>300$ & $\begin{array}{l}\text { Terminalia chebula Retz. (Terminalia chebula Retz., Terminalia chebula Retz. var. tomentella } \\
\text { (Kurz) C. B. Clarke), Carthamus tinctorius L., Aucklandia lappa Decne. }\end{array}$ & $\begin{array}{l}3 / 3 \\
(100 \%)\end{array}$ \\
\hline $201 \sim 300$ & $\begin{array}{l}\text { PHYLLANTHI FRUCTUS, Amomum kravanh Pierre ex Gagnep, Punica granatum L., } \\
\text { Zha-Xun, Piper longum L, Ewgewia caryophyllata Thunb., MOSCHUS (Moschus } \\
\text { berezovskii Flerov, Moschus moschiferus Linnaeus, Mochus sifanicus Buchner), } \\
\text { Inula racemosa Hook.f., Myristica fragrans Houtt. }\end{array}$ & $\begin{array}{l}9 / 9 \\
(100 \%)\end{array}$ \\
\hline $99 \sim 200$ & $\begin{array}{l}\text { Calcitum, BOVIS CALCULUS, Adhatoda vasica Nees, CINNAMOMI CORTEX, Aquilaria } \\
\text { agallocha Roxb (Aquilaria sinensis (Lour.) Gilg, Aquilaria agallocha Roxb), Zingiber } \\
\text { officinale Rosc., Glycyrrhiza uralensis Fisch. (Glycyrrhiza uralensis Fisch., Glycyrrhiza } \\
\text { inflata Bat.), Amomum tsaoko Crevost et Lemair, Styrax benzoin Dryand., Tinospora } \\
\text { sinensis (Lour.) Merr. }\end{array}$ & $\begin{array}{l}10 / 19 \\
(53 \%)\end{array}$ \\
\hline $51 \sim 100$ & $\begin{array}{l}\text { BAMBUSAE CONCRETIO SILICEA (Bambusa textilis McClure and Schizostachyum } \\
\text { chinense Rendle root exudates), Dalbergia odorifera T. Chen, OLIBANUM, Rubia } \\
\text { cordifolia L., Selenarctos thibetanus G. Cuvier, Rhodiola crenulata(Hook. f. et Thoms.) } \\
\text { H. Ohba } \\
\text {, Acorus calamus L., Aconitum pendulum Busch, Piper nigrum L., Crocus sativus L., } \\
\text { Malva verticillata, Abelmoschus moschatus Medic, Cassia obtusifolia L., Tribulus terrestris L. }\end{array}$ & $\begin{array}{l}14 / 19 \\
(73 \%)\end{array}$ \\
\hline $11 \sim 50$ & $\begin{array}{l}\text { FERULAE RESINA (Ferula fukanensis K. M. Shen, Ferula sinkiangensis K. M. Shen), Kaempferia } \\
\text { galanga L., Gossampinus malabarica (DC.) Mern, Cinnabaris, MARGARITA (Cristaria plicata } \\
\text { (Leach), Hyriopsis cumingii (Lea)., Pinctada martensii (Dunker)), Borax, Corallium }\end{array}$ & $\begin{array}{l}7 / 103 \\
(7 \%)\end{array}$ \\
\hline
\end{tabular}


Table 9 Shared-use medicines involved in TCM prescriptions

\begin{tabular}{llll}
\hline Medicinal materials name & Scientific name & Usage frequency & Use frequency ranking in the original text [24] \\
\hline Glycyrrhizae Radix et Rhizoma & Glycyrrhiza uralensis Fisch., Glycyrrhiza inflata Bat. & $65.35 \%$ & 1 \\
Fritillaria & Fritillaria cirrhosa D.Don & $14.20 \%$ & 11 \\
Paeoniae radix rubra & Paeonia veitchii Lynch & $9.76 \%$ & 27 \\
Aquilariae Lignum Resinatum & Aucklandia lappa Decne. & $6.82 \%$ & 48 \\
\hline
\end{tabular}

used by both medicinal systems to treat digestive diseases, which indicated a high consensus of using volatile compounds to warm the stomach and promote digestion in TM and TCM.

\section{The family and genus characteristics of shared-use medicines used in TM and TCM}

In the present article, we have listed a total of 136 shared-use medicines in TM and TCM, of which angiosperm, belonging to 53 families and 101 species, was used the most, accounting for $71 \%$ of the total 136 types of shared-use medicines. Leguminosae was the most widely used family, followed by Zingiberaceae, Umbelliferae, Compositae, Liliaceae, Polygonaceae, Labiatae, Thymelaeaceae, and Ranunculaceae. Three pteridophytes (having two families and three species) were used in both TM and TCM. Sixteen animal resources and 12 mineral resource medicines were used, accounting for $12 \%$ and $9 \%$, respectively, of the total. The high proportion is due to the wide use of animal and mineral resource medicines in TM. Detailed information was given in Table 11.

\section{Discussion}

It is interesting and complicated to discuss the relationships between the two neighboring traditional systems of medicine and their long histories. Naive materialism is the foundation of both; meanwhile, TM is largely influenced by Tibetan Buddhist culture while TCM is largely influenced by Confucian culture. In terms of medical theory and knowledge on medicinal materials, the two-, three-, five-, and six-group methods are widely used in both TM and TCM, such as the "five essences" (water, fire, soil, qi, air) of the former and the "five elements" (gold, wood, water, soil, fire) of the latter. There is a high similarity in knowledge of the two medicinal systems on properties and flavors of medicine; for example, both styles of medicine are grouped according to the properties of "cold and warm," and "sour, bitter, sweet, pungent, and salty" are used both in the "six flavors theory" of TM and the "five flavors theory" of TCM. However, the present paper discussed the relationship of TM and TCM in terms of the similarity and dissimilarity of efficacy and the origins of shared-use medicines, instead of the philosophical theory.

The possible reasons for the similarity of different medical systems in the usage of medicine could be the following:

1. Significant pharmacological effects of medicine;

2. Mutual communication between the two medical systems about clinical practices;

3. Same influence of other traditional systems of medicine;

4. Coincidence.

While the reason for different medicinal properties of the same medicine varies, it could be due to the following:

1. Different medicinal parts or preparation used;

2. Different medicinal prescriptions;

3. Distinctive local culture and heritage of knowledge on medicinal application;

4. Diverse regional common ailments as well as natural and socioeconomic conditions;

Table 10 Diseases groups according to medicinal property using shared-use medicines in TCM and TM

\begin{tabular}{|c|c|c|}
\hline Disease group & Number of TCM involved & Number of TM involved \\
\hline Circulatory system diseases & 12 & 13 \\
\hline Urinary system diseases & 18 & 13 \\
\hline Immune system diseases & 21 & 31 \\
\hline Nervous system diseases & 23 & 16 \\
\hline Reproductive system diseases & 24 & 21 \\
\hline Motion system diseases & 24 & 14 \\
\hline Respiratory system diseases & 36 & 34 \\
\hline Digestive system diseases & 61 & 62 \\
\hline
\end{tabular}


Table 11 Number of species used in shared-use medicines

\begin{tabular}{lll}
\hline Classification & Family & No. of species used \\
\hline Angiosperm & Leguminosae & 9 \\
& Zingiberaceae & 8 \\
& Apiaceae & 7 \\
& Asteraceae & 6 \\
& Liliaceae & 4 \\
& Polygonaceae & 4 \\
& Labiatae & 3 \\
& Thymelaeaceae & 3 \\
Gymnosperm & Ranunculaceae & 3 \\
& Others & 54 \\
Resinae & Polypodiaceae & 2 \\
Animalia & Selaginellaceae & 1 \\
Mineral group & - & 5 \\
Others & - & 16 \\
\hline
\end{tabular}

Based on the research results, this article has drawn the following inferences.

\section{Medicinal knowledge exchange occurred during the parallel development of TM and TCM}

\section{Shared-use medicines are mostly determined by flora similarity and medicinal trade}

It can be observed in the Table 12 that a total of 67 shared-use medicines were distributed in both the $\mathrm{Ti}$ betan and Han regions, accounting for $49 \%$ of the total. The Huaxia people originated in northwestern China, rising north of the Yangtze River and belonging to the China-Japan forest subregion. While the Tibetans rose in the Qinghai-Tibetan Plateau, where the eastern part belongs to the China-Himalayan ecological subregion, and the west is the Qinghai-Tibetan Plateau plant subregion. There is a large crossover in plant varieties between the two ethnic regions [25]. In comparing the shared-use medicines listed in Table 9 with common plants in Northern China [26] and the Tibetan regions [27], Compositae, Leguminosae, Gramineae, Ranunculaceae, Labiatae, Umbelliferae, Liliaceae, and Rosaceae are common families within both regions. Therefore, the large number of cross-plant species formed by flora is the main reason for the large number of shared-use medicines in the two traditional systems of medicine.

As shown in the Table 12, 56 taxa of shared-use medicines were imported from the non-Han region to the Tibetan region, accounting for $35 \%$ of the total. On the other hand, 20 taxa were imported from the non-
Tibetan region to Han inland, accounting for $15 \%$ of the total. This suggests that imported medicinal materials from the southern regions have some influence on the two medicinal systems, especially on TM (from Table 7), and that both TM and TCM have closely communicated with other extraterritorial medical systems and acquired practical experience regarding the usage of medicine.

\section{The shared-use medicines marked significant differences in their medicinal properties}

In the tang dynasty, the central plains and Tubo exchanged information closely, according to the edited books and research articles. It is generally believed that TM has been largely influenced by TCM, which is reflected in the pulse diagnosis and visceral knowledge of TM [28]. However, this paper showed that shared-use medicines used in TM and TCM marked significant differences in their medicinal properties, and most of the widely used Tibetan medicines were imported from the non-Han area. The unique medicinal use of TM is reflected in earlier books, such as Yutu Materia Medica, Tara Materia Medica, and Miaoyin Materia Medica. Based on the analysis, this article believed that medicinal materials and medicinal use experiences of TM were mainly summarized by its clinical practitioners during and before the Tubo dynasty in the extreme natural conditions in the Tibetan plateau. TM developed in parallel with TCM and was greatly influenced, especially in medicinal resources by traditional medicinal systems in southern Asia in the later stage.

As for the shared-use medicines distributed in the $\mathrm{Ti}$ betan plateau listed in the Table 12, although they are local products, varieties such as Notopterygium incisum Ting ex H. T. Chang, Gentianae Macrophyllae Radix, Moschus, and Gansong, were recorded in the ancient traditional Chinese medicine and were traded to the Han region through the western Sichuan Plateau, which was controlled for a long time by the Central Plains dynasticism since the Qin and Han dynasties [29], as well as the southern Gansu province. For example, the use of Snow Lotus Herb [30] can be traced back to Qing dynasty in Supplements to Compendim of Materia Medica, which appears to be a teaching from Uygur Medicine, while the use of Arenaria kansuensis Maxim has been recorded in The Collection of Chinese Herbal Medicines 1975 version, which appears to be a teaching from folk practices. Therefore, the Tibetan plateau serves as a medicinal resource for TCM.

In summary, shared-use medicines by TM and TCM marked a significant difference in medicinal properties.

2. Shared-use medicines with similar medicinal properties presented an obvious commercial 


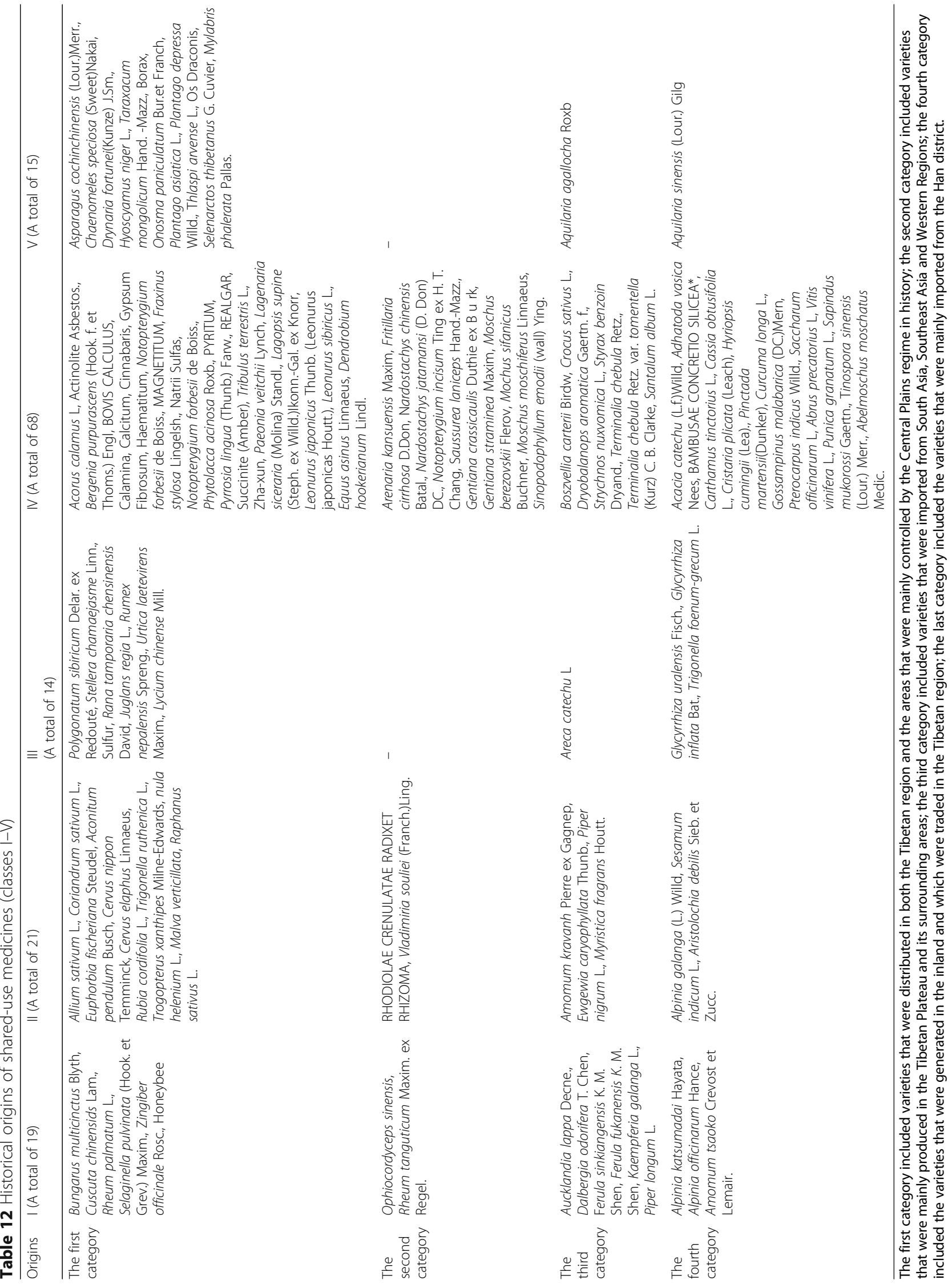


characteristic of materia medica as well as the same chemical profile

It can be observed in Table 7 that, out of 19 shareduse medicines with similar medicinal effects (class I), a number of 13 were traded medicines, up to $68 \%$ of the total, which was much higher than other proportions. These varieties have marked an obvious commodity attribute. For example, RHEI RADIX ET RHIZOMA was commonly traded through the silk road. Ophiocordyceps sinensis was the representative example of traded medicine from the Tibetan region to Han inland, which was originally recorded in the ancient Tibetan medicinal book Ten Million Buddhist Relics by Suka.Nii6anmuduoji (1439-1475). It was not traded in the Han region until the Kangxi period and was recorded in A General Description of SiChuan in the Yongzheng period. Rhodiola crenulata (Hook. f. et Thoms.) H. Ohba was recorded in Chinese Pharmacopoeia in the year of 1977 as commonly used Tibetan medicine, while currently it is widely used by traditional Chinese medicine.

As shown in the Table 12, among the shared-use medicines that are used in TM and TCM, there was a large number of aromatic medicines that are rich in volatile compounds. Those aromatic medicines are used to warm the stomach and promote digestion in both TM and TCM, which is closely related to the pharmacological activity of volatile components.

\section{Tibetan plateau not only provides medicinal usage knowledge of TCM, but it also serves as a supply of medicinal resources attributing to "high altitude" locations} Tibetan culture exchanged medicinal information closely with Han culture officially during the Tang dynasty. After the perdition of the Tubo regime, the culture exchange moved towards the flock through the tea-horse ancient road. Medicines as Ophiocordyceps sinensis, Rhei Radix Et Rhizoma, Nardostachys chinensis Batal., Gentianae Macrophyllae Radix, Fritillaria cirrhosa D. Don, Notopterygium incisum Ting ex H. T. Chang, and Moschus were imported from the Tibetan plateau and were widely used in TCM. It can be observed in the Table 12 that a number of 16 traditional Chinese medicines were imported from the Tibetan plateau, which was much higher than the Tibetan medicines that were imported from the Han region (five species). The traditional Chinese medicines that were imported from the Tibetan plateau could be divided into the following three categories: the first category, such as Notopterygium incisum Ting ex H. T. Chang, Fritillaria, Moschus, and Gentianae Macrophyllae Radix, after a long history of medicinal use in TCM, were used quite differently in TM; the second category, such as Ophiocordyceps sinensis and Rhodiolae Crenulatae Radixet Rhizoma, were used similarly in TM and TCM, because they were imported during the near ancient time or even in modern times; the last category, such as the Snow Lotus Herb, presented a different medicinal effect from TM, because of the influence of other traditional medicinal systems. In summary, the Tibetan plateau not only provides medicinal usage knowledge of TCM, but it also serves as a supply of medicinal resources. According to the literature, the western Sichuan and western Yunnan regions were the main trade routes. In contrast, few Tibetan medicines were imported from the Han region, and no available ancient literature could provide a clue that TM has learned knowledge from TCM. Therefore, this paper believed that TM started to acquire medicinal knowledge from TCM only in modern times. For example, Fritillaria cirrhosa D. Don has been used in TM to stop coughs, as it is used in TCM, only since modern times.

Attributed to its unique geographical location and cultural diversity, the Tibetan region plays a role as a development cradle for various traditional medical theories and knowledge

Tibetan culture has been extensively and deeply influenced by many ancient civilizations of the world. It has been exchanging knowledge with ancient Indian, Central Plains and Persian cultures for a long time. During the Hellenistic period after Alexander's expedition, it was inevitably influenced by the Mediterranean through ancient India. There are not many existing cultures like Tibetan culture that are influenced by multi-mainstream cultures from the ancient world. It also shows a stark contrast to the demised history of various cultures in the neighboring Western Regions.

Due to the religious influences and relatively closed environment, the social development of areas in the Tibetan region has been slow since the "Peihong period" of Tibetan Buddhism, thus providing conditions for the preservation and inheritance of medicinal knowledge. Taking the Terminalia chebula Retz., which is used most commonly in TM, as an example, its prescription "Da San Guo Tang" came from India and is still commonly used in a Tibetan compound recipe. In contrast, after the Ming dynasty, Terminalia chebula Retz. var. tomentella (Kurz) C. B. Clarke and Phyllanthi Fructus were almost discarded from use in TCM prescriptions. Tibetan medicine Zota and some other varieties are similar examples of this case. Therefore, we believe the medicinal distinctness of the Tibetan plateau has been preserved since the "Xiang Xiong" period, and foreign medicines, including Terminalia chebula Retz., Aucklandia lappa Decne., and Carthamus tinctorius L., have also been promoted in TM. This can be demonstrated by the special status of exotic species in Tibetan medicinal prescriptions. 
Table 13 Shared-use medicines that are involved in more than 51 TM preparations

\begin{tabular}{ll}
\hline Efficacy classification & Shared-use medicines \\
\hline Class I & Aucklandia lappa Decne., PHYLLANTHI FRUCTUS, Alpinia katsumadai Hayata, Piper longum L, \\
& Amomum tsaoko Crevost et Lemair, Dalbergia odorifera T. Chen, Rhodiola crenulata \\
& (Hook. f. et Thoms.) H. Ohba, Piper nigrum L. \\
Class II & Myristica fragrans Houtt., ZINGIBERIS RHIZOMA, Ewgewia caryophyllata Thunb., Rubia cordifolia L., \\
& Malva verticillata. \\
Class III & Glycyrrhiza uralensis Fisch., Glycyrrhiza inflata Bat.. \\
Class IV & Terminalia chebula Retz., Carthamus tinctorius L., MOSCHUS (Moschus berezovskii Flerov, Moschus \\
& moschiferus Linnaeus, Mochus sifanicus Buchner), Calcitum, BOVIS CALCULUS, Adhatoda vasica Nees, \\
& CINNAMOMI CORTEX, Punica granatum L., Styrax benzoin Dryand., Tinospora sinensis (Lour.) Merr., \\
& BAMBUSAE CONCRETIO SILICEA (Bambusa textilis McClure and Schizostachyum chinense Rendle \\
& root exudates), OLIBANUM, Selenarctos thibetanus G. Cuvier, Aconitum pendulum Busch, Crocus \\
& sativus L., Abelmoschus moschatus Medic, Cassia obtusifolia L., Tribulus terrestris L. \\
& Aquilaria agallocha Roxb \\
\hline Class V &
\end{tabular}

Research results of the present study are scientific and representative

To verify the scientific research results, this study also compared 3107 TM varieties and more than 2200 kinds of TCM varieties according to the Dictionary of Chinese National Medicine and The Collection of Chinese Herbal Medicines. A total of 313 shared-use medicines were documented, and the proportions of each variety are $12 \%$ for class I, $12 \%$ for class II, $12 \%$ for class III, $60 \%$ for class IV, and $50 \%$ for class $\mathrm{V}$, which was consistent with the results of this paper, thus indicating the representativeness of the statistical results of this study (Table 13).

\section{Conclusions}

The present study was based on the statistical analysis of the authoritative publications and papers of national medicine and traditional Chinese medicine. To reflect the exact clinical use status of TM and TCM and to make sure the results are representative, clinical formulas of TM were used for statistics.

Studies showed that medicinal knowledge exchange occurred during the parallel development of TM and TCM, the shared-use medicines are mostly determined by the flora similarity and medicinal trade and marked significant differences in their medicinal properties, and shared-use medicine with similar medicinal properties presented an obvious commercial characteristic of materia medica, as well as the same chemical profile. From the breed point of view, Tibetan plateau not only provides medicinal usage knowledge of TCM, but it also serves as a supply of medicinal resources, attributing to the "high altitude" influence. Attributed to its unique geographical location and cultural diversity, the Tibetan region plays a role as a development cradle for various traditional medical theories and knowledge.

\section{Abbreviations}

TCM: Traditional Chinese medicine; TM: Tibetan medicine

\section{Acknowledgements}

We are grateful to the administrative agencies of Guo-yue Zhong for his help while carrying out this study.

\section{Authors' contributions}

$\mathrm{MZ}$ is involved in the study design, literature and vouchers review, systematization and analysis of the data, and wrote the first draft. KW is involved in the study and revised and finalized the manuscript. RG conceived and designed the study and supervised the whole work. SZ confirmed the drug efficacy in the table. All authors read and approved the final manuscript.

\section{Funding}

This work was financially supported by the National Natural Science Foundation of China (83571699) and the fourth national survey on Chinese material medica resources.

Availability of data and materials Not applicable.

Ethics approval and consent to participate

Not applicable.

\section{Consent for publication}

Not applicable.

\section{Competing interests}

The authors declare that they have no competing interest.

\section{Author details}

${ }^{1}$ Chengdu University of Traditional Chinese Medicine, No. 1166, Liu-tai Road, Wenjiang District, Chengdu 611173, China. ${ }^{2}$ Institute of Chinese Medicinal Science, State Key Laboratory of Quality Research in Chinese Medicine, University of Macau Avenida da Universidade, Taipa, Macau, China. ${ }^{3}$ Chengdu Medical College, No. 783, Xing-du Road, Xingdu District, Chengdu 610083,

China

Received: 12 March 2019 Accepted: 28 July 2019

Received: 12 March 2019 Accepted: 28 July

\section{References}

1. Zhong G-Y, Zhou F-C, Shi S-M, et al. Actuality investigation on general crude drugs and its quality standard of Tibetan medicine. China Journal of Chinese Materia Medica. 2012:37(16):2349-54.

2. Cai J-F. General history of Tibetan medicine. Sining: Qinghai Peolple's Publishing House; 2002

3. Mao J-Z. Basic theories of Tibetan medicine: Gansu Ethnic Publishing House; 1999.

4. Li L-S. Research on the Intersection of Cultural Elements in the Chinese Medicine and Tibetan Medicine. World I Integrated Traditional Western Med. 2016;11(1):1-5. 
5. Company of Chinese Medicinal Materials. Journal of Chinese traditional medicine resources: Science Press; 1994.

6. Jia M-R. Dictionary of Chinese national medicine: China Medical Science Press; 2016.

7. Ministry of Health of the People's Republic of China. Pharmaceutical standards of the ministry of health of the People's Republic of China. Beijing: People's Publishing House; 1995.

8. Sanitary Bureau of Tibet, Qinghai, Szechwan, Gansu, Yunnan, Sinkiang. Tibetan medicine standards for six provinces. Sining: Qinghai Peolple's Publishing House; 1978.

9. Writing Group of the Collection of Chinese Herbal Medicines. The collection of Chinese herbal medicines. Beijing: People's Publishing House; 1975.

10. Chinese Pharmacopoeia Commission, Chinese Pharmacopoeia, Book 1. China Medical Science Press ; 2015.

11. Gongbu YY. The four-part medical classics. Ma S-L, Luo D-S. et al. translation and annotation. Shanghai: Shanghai Scientific \& Technical Publishers; 1987.

12. Dimaer Danzhenpengcuo. Crystal beads (Chinese version). Shanghai: Shanghai Scientific \& Technical Publishers; 1986.

13. Northwest Plateau Institute of biology, Chinese Academy of Sciences. Tibetan Medicine Chronicles. Sining: Qinghai Peolple's Publishing House; 1991.

14. Chinese Herbalism Editorial Board, State Administration of Traditional Chinese Medicine of the People's Republic of China 1. Chinese herbalism. Chinese Materia Medica (Book Tibetan Medicine). Shanghai: Shanghai Scientific \& Technical Publishers; 2002.

15. Pharmaceutical Inspection in Qinghai Province, Qinghai Institute of Tibetan Medicine. Tibetan medicine in China (Book 1 3). Shanghai: Shanghai Scientific \& Technical Publishers; 1969.

16. Luo D-S. Tibetan herbals in China. Beijing: THE ETHNIC PUBLISHING HOUSE; 2012.

17. Editorial Committee of. Flora Reipublicae Popularis Sinica from Chinese Academy of Sciences. Flora Reipublicae Popularis Sinica. Beijing: Science Press; 2004.

18. Fu L-G, Cheng T-Q, Lang K-Y, et al. Higher plants of China. Qingdao: Qingdao Publishing House; 2002.

19. Luo D-S. Revised crystal beads. Sichuan Scientific \& Technical Publishers; 2004.

20. Jiangsu New Medical College. Chinese Medicine Dictionary. Shanghai: Shanghai Scientific \& Technical Publishers; 1986

21. Gawu. Tibetan medicine crystal mirror materia medica: Tibetan. THE ETHNIC PUBLISHING HOUSE; 1995.

22. Xing Z-G, Hu X-M, Zhao D-F. Medicinal Mineral of Qinghai and Tibet . Qinghai People's Publishing House; 1985.

23. Ministry of Health Inspection Institute for Pharmaceutical and Biological Products, Yunnan Pharmaceutical Inspection Institute. Chinese ethnic medicine ethnography, Book 1. People's Medical Publishing House; 1984.

24. Xi P, Tang H-M, Qian $L$, et al. Investigation on the prescription of traditional Chinese medicine in outpatient clinic. Clinical Pharmacy. 2008;18(3):61-4.

25. Li H, Li S-G. The floristic structure of East Xizang region. Geogr Res. 1984; 8(2):64-70.

26. Wang H-S. The evolution and sources of North China's flora. Acta Geographica Sinica. 1999:54(3):213-23.

27. Wu Z-Y. Flora of Tibet. Book5. In: Science Press; 1987.

28. Cai J-F. General history of Tibetan medicine: Qinghai Peolple's Publishing House; 2002. p. 81 86

29. Hu X-X. Handbook of economic plant: Science Press; 1955. p. 354.

30. Li J-S. A study on the resources of snow lotus herb -- medicinal plants of Saussurea in China. China Union Medical College; 1999.

\section{Publisher's Note}

Springer Nature remains neutral with regard to jurisdictional claims in published maps and institutional affiliations.

\section{Ready to submit your research? Choose BMC and benefit from}

- fast, convenient online submission

- thorough peer review by experienced researchers in your field

- rapid publication on acceptance

- support for research data, including large and complex data types

- gold Open Access which fosters wider collaboration and increased citations

- maximum visibility for your research: over $100 \mathrm{M}$ website views per year

At BMC, research is always in progress.

Learn more biomedcentral.com/submissions 